\title{
Commercial Film Detection and Identification Based on a Dual-Stage Temporal Recurrence Hashing Algorithm
}

\author{
Xiaomeng $\mathrm{Wu}$ \\ National Institute of \\ Informatics, 2-1-2 \\ Hitotsubashi, Chiyoda-ku, \\ Tokyo 101-8430, Japan \\ wxmeng@nii.ac.jp
}

\author{
Narongsak Putpuek ${ }^{*}$ \\ Chulalongkorn University, \\ Bangkok 10330, Thailand \\ narongsak.p@ \\ student.chula.ac.th
}

\author{
Shin'ichi Satoh \\ National Institute of \\ Informatics, 2-1-2 \\ Hitotsubashi, Chiyoda-ku, \\ Tokyo 101-8430, Japan \\ satoh@nii.ac.jp
}

\begin{abstract}
This paper proposes a dual-stage temporal recurrence hashing algorithm for fully unsupervised and super-fast Commercial Film (CF) mining in large-scale broadcast video archives. The first-stage hashing algorithm converts a large amount of video segments into a set of temporal occurrence patterns on the basis of a luminance-based fingerprinting strategy. During the second stage, each temporal recurrence of a certain segment is mapped to an inverted index to build a recurrence hashing histogram, which is the key idea in this paper to achieve super-fast CF detection and identification. The detection and identification task is then converted into one of detecting local maximums from this hashing histogram, with one local maximum corresponding to one pair of two identical CF segments. A large-scale archive, containing a 10-hour and a 1-month sequence, was used for experimentation. Our algorithm performed CF detection and identification on the 1-month sequence in merely $\mathbf{8 7}$ minutes and had $90.59 \%$ detection accuracy and $98.06 \%$ localization accuracy.
\end{abstract}

\section{Categories and Subject Descriptors}

H.3.1 [Information Storage and Retrieval]: Content Analysis and Indexing - indexing methods; H.2.8 [Database Management]: Database Applications-data mining

\section{General Terms}

Algorithms, Experimentation

\section{Keywords}

Commercial film, data mining, repetition-oriented, hashing algorithm

\section{INTRODUCTION}

In recent years, Commercial Film $(\mathrm{CF})$ mining has attracted the attention of researchers. This task aims at de-

\footnotetext{
${ }^{*}$ This work was performed when Narongsak Putpuek was
} visiting National Institute of Informatics as an intern.

Permission to make digital or hard copies of all or part of this work for personal or classroom use is granted without fee provided that copies are not made or distributed for profit or commercial advantage and that copies bear this notice and the full citation on the first page. To copy otherwise, to republish, to post on servers or to redistribute to lists, requires prior specific permission and/or a fee.

Copyright 20XX ACM X-XXXXX-XX-X/XX/XX ...\$10.00. veloping techniques for detecting, localizing, and identifying CFs broadcasted on different channels and time slots. There have been a large number of promising applications using CF mining techniques to solve real-world problems, including broadcast media analysis, market research, and $\mathrm{CF}$ management. CF mining techniques can be categorized into two domains, knowledge-based and repetition-based. In this paper, we propose a novel repetition-based technique, which aims at both generic and efficient $\mathrm{CF}$ mining. The purpose and the main characteristics of this technique are as follows. 1) We aim at fully unsupervised CF mining, i.e. there is neither a training database nor reference $\mathrm{CF}$ database provided beforehand. 2) We aim at more generic CF mining compared with knowledge-based studies. Only one type of prior knowledge, i.e. the restricted duration of the CF segment, is used for noise filtering. 3) We assume that the $\mathbf{C F}$ boundaries are unknown, i.e. we aim at automatically achieving all capabilities, including detection, localization, and identification. 4) We aim at super-fast CF mining. Our algorithm can perform CF detection and identification on a 10-hour sequence within four seconds. 5) We evaluated our algorithm on a large-scale broadcast video archive containing a 1-month sequence. $\mathrm{CF}$ detection and identification of this sequence took only 87 minutes.

\section{RELATED WORK}

Knowledge-based CF detection techniques [1, 5, 8, 10, 12, 13] focus on the intrinsic characteristics of CFs and use them as a certain type of prior knowledge for detecting CFs. The criteria or features of these works are based on the prior knowledge including monochrome frame [5, 8, 12, 13], silence frame $[5,8,10]$, product frame $[1,5]$, duration [12], shot information (e.g. frequency or style of cuts, etc.) $[1,8$, $10,13]$, and motion information (e.g. edge change ratio, motion vector length, etc.) $[8,10,12,13]$.Knowledge-based methods are normally more efficient than repetition-based techniques, but most are not generic enough because of the data-dependent prior knowledge they use.

Because a single TV advertisement can be broadcast repeatedly over the course of weeks, months, and even years, CFs can be regarded as a certain type of repetition in the broadcast video stream. Some recent studies $[2,3,4,6,7$, $9,11,14]$ have tried to use this property to detect and identify CFs. Some of these studies $[4,6,7,11,14]$ are based on image hashing, in which all descriptors (i.e. all frames) of the whole video stream are mapped to an inverted index to build a hash table. Recurring CF segments are then detected and identified by searching for frames with the same hash value. To tackle the problem of the large computational burden caused by handling all descriptors, Berrani et al. [2] proposed a shot-based technique that focuses on representative frames instead of the whole video stream. In 
this study, clustering is used instead of hashing to group representative frames into many small clusters. These clusters are further grouped on the basis of the similarity of the temporal distribution of cluster members (i.e. representative frames in a certain cluster) to accommodate possible asynchronous selection of representative frames. Other studies $[3,9]$ focus on CF mining based on acoustic or visual and acoustic information. Repetition-based techniques are more generic than knowledge-based ones, but cause a larger computational burden.

\section{OVERVIEW}

Given a broadcast video stream as the input, our target is to output a set of CF clusters. One CF cluster corresponds to one specific CF, e.g. CF of Apple Macintosh. Each CF cluster contains multiple identical CF segments. One CF segment corresponds to one instance of a specific CF. CF segments within the same CF cluster are normally broadcasted on different channels and time slots. These CF segments range in length from a few seconds to several minutes.

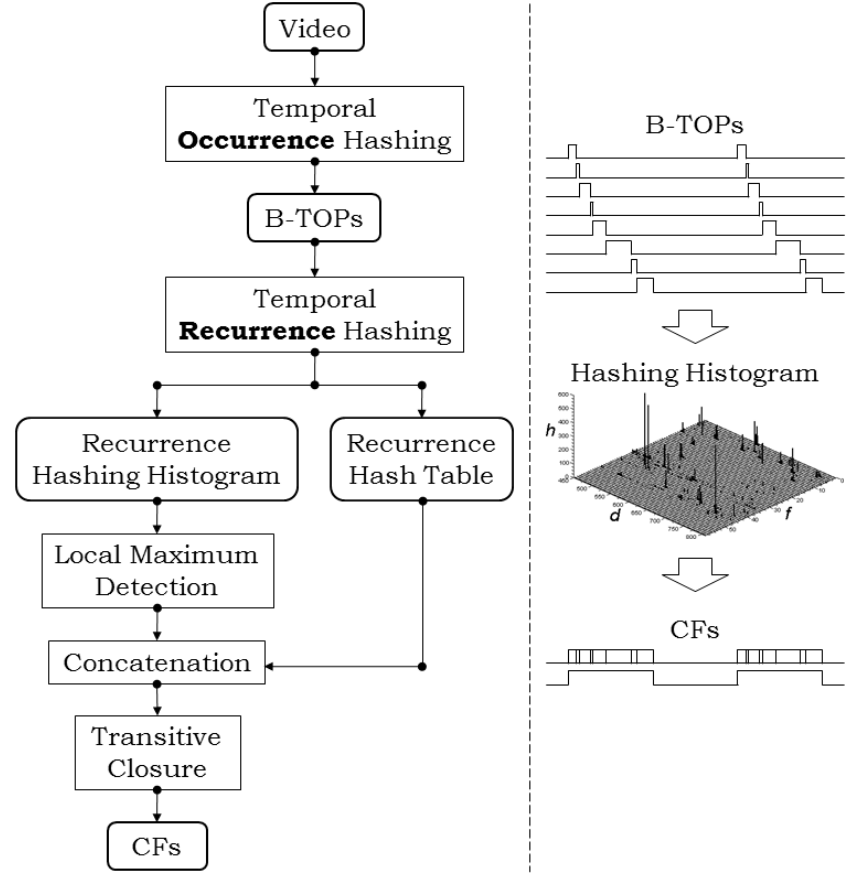

Figure 1: Framework of the CF detection and identification system.

A dual-stage temporal recurrence hashing algorithm is proposed for super-fast CF detection, localization, and identification. The framework of this algorithm is shown in Figure 1. The first-stage hashing algorithm converts a video into a set of binary sequences, each of which represents the existence of discrete short segments with a specific fingerprint. The second-stage hashing algorithm converts the set of binary sequences into another hash table on the basis of the temporal distribution of short segment pairs, and a hashing histogram is built as the result. Via histogram voting, the consecutive short segments of the same CF are merged, and each local maximum in this histogram corresponds to a specific pair of identical CF segments. Based on each local maximum, we can detect the existence of CFs, assemble the discrete short segments into a whole CF segment, and further detect the pairwise relationship between identical $\mathrm{CF}$ segments. Instead of brute-force pairwise matching, this strategy applies hashing to both segment matching and segment assembling so that the algorithm only requires a single scan of the corresponding data. As a result, the algorithm can achieve far faster CF mining than other related studies.

\section{DUAL-STAGE TEMPORAL RECURRENCE HASHING ALGORITHM}

\subsection{Temporal Occurrence Hashing}

A luminance-based fingerprinting strategy is used in this paper, because of its simplicity and effectiveness. Each video frame is first normalized by Eq. 1 , where $I_{x, y}$ and $I_{x, y}^{*}$ indicate the luminance value of each pixel $(x, y)$ before and after normalization. $\mu$ and $\sigma$ are the mean and the standard deviation defined by Eq. 2 and 3, where $n_{I}$ is the number of pixels.

$$
\begin{gathered}
I_{x, y}^{*}=\frac{I_{x, y}-\mu}{\sigma} \\
\mu=\frac{\sum I_{x, y}}{n_{I}} \\
\sigma=\sqrt{\frac{\sum\left(I_{x, y}-\mu\right)^{2}}{n_{I}}}
\end{gathered}
$$

The normalized frame is then divided into $16(4 \times 4)$ blocks. The first and the second luminance moment, i.e. the mean and the standard deviation of $I_{x, y}^{*}$, are calculated for each block. Thus, a 32-dimensional feature vector is generated in which the first 16 dimensions correspond to the first moment and the second 16 dimensions correspond to the second moment. This feature vector is further binarized into a 32-bit fingerprint by comparing each component with the mean of the corresponding moment. A video sequence is denoted by $\mathbf{I}=\left\{I_{1}, I_{2}, \cdots, I_{n}\right\}$, where $I_{i}$ indicates the $i$ th frame and $n$ is the number of frames. A fingerprint set $\mathbf{F}=\left\{F_{1}, F_{2}, \cdots, F_{n}\right\}$ is then generated from $\mathbf{I}$, with $F_{i}$ being the 32-bit fingerprint of $I_{i}$.

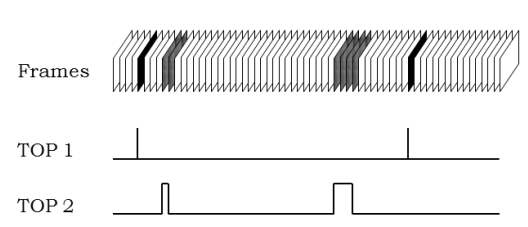

(a) Example of B-TOPs.

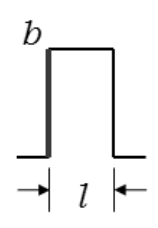

(b) Peak.
Figure 2: B-TOP representation.

All frames in $\mathbf{I}$ are inserted into a hash table regarding the corresponding 32-bit fingerprint as the hash value. A Temporal Occurrence Pattern is generated from each bucket of the hash table and named B-TOP (Bucket-TOP). B-TOP is a binary sequence representing the existence of frames in this bucket (Figure 2a). All B-TOPs have the same length $n$ (number of frames). Suppose that a bucket contains $k$ frames, then the corresponding B-TOP will contain $k$ nonzero values at the temporal positions of these $k$ frames, and all other $(n-k)$ values will be zero. B-TOP can also be represented as a set of peaks. A peak is a short segment composed of one or multiple frames with the same fingerprint. One peak corresponds to one temporal occurrence of a specific fingerprint. These peaks range in length from one frame to several seconds. Here, a peak $p_{i}$ will be represented by two parameters $p_{i} \rightarrow b_{i}, l_{i}$, with $b_{i}$ being the temporal position of the beginning frame and $l_{i}$ being the length of the peak (Figure $2 \mathrm{~b}$ ). Thus, a B-TOP $\mathbf{p}$ can be represented as $\mathbf{p}=\left\{p_{1}, p_{2}, \cdots, p_{m}\right\}$, with $m$ being the number of peaks. 


\subsection{Temporal Recurrence Hashing}

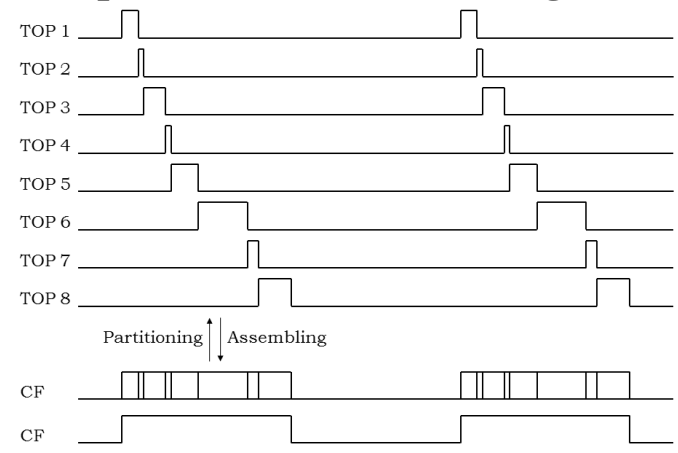

Figure 3: TOPs derived from two identical segments.

Figure 3 shows an example of B-TOPs derived from a pair of identical CF segments. Each segment has been discretely partitioned into eight peaks. These peaks need to be automatically assembled to form the whole segment. From Figure 3, we found that the temporal distribution of these peak pairs, i.e. the position of and the interval between the two peaks in each pair, are similar to each other. The problem is how to automatically assemble these peaks. One solution is to define a similarity between B-TOPs based on the temporal distribution and then apply brute-force pairwise matching or clustering to all B-TOPs. However, this solution has a large computational burden because of the large number and the large size of B-TOPs. Instead, we propose to use a hash function to convert the temporal distribution of each peak pair into a hash value so that the peak assembling problem can be solved on the basis of the hash table. As a result, the algorithm can avoid the time-consuming computation due to the brute-force pairwise matching between B-TOPs.

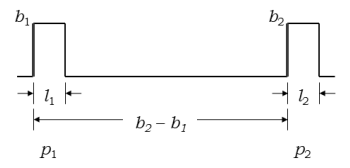

Figure 4: Representation of a peak pair $\left\{p_{1}, p_{2}\right\}$.

The temporal recurrence hashing algorithm regards the peak pair as the basic unit. Each peak pair $\left\{p_{i}, p_{j}\right\}$ with $i<$ $j$ is represented by two parameters, $f_{i, j}$ and $d_{i, j}$ (Figure 4 ), respectively defined by Eq. 4 and Eq. $5 . f_{i, j}$ indicates the temporal position of the former peak $p_{i}$, and $d_{i, j}$ indicates the interval between $p_{i}$ and $p_{j}$.

$$
\begin{gathered}
f_{i, j}=b_{i} \\
d_{i, j}=b_{j}-b_{i}
\end{gathered}
$$

One peak pair corresponds to one temporal recurrence of a specific fingerprint. Based on $f$ and $d$, all peak pairs are mapped to an inverted index and inserted into a bin of a 2dimensional hash table (Figure 5). The resolution of $f$ and $d$ are respectively set to minute and second. The purpose of this setting is to ensure that the peak pairs derived from two identical CF segments, the length of which is assumed to be less than one minute, can be inserted into the same bin or at least neighboring bins. For all B-TOPs, we calculate $f$ and $d$ from all peak pairs in each B-TOP, and then vote them to the histogram. Given a peak pair $\left\{p_{i}, p_{j}\right\}$, the vote value is calculated by Eq. $6 . h\left(f_{i, j} ; d_{i, j}\right)$ can be regarded as the number of overlapping frames in each bin. Theoretically,

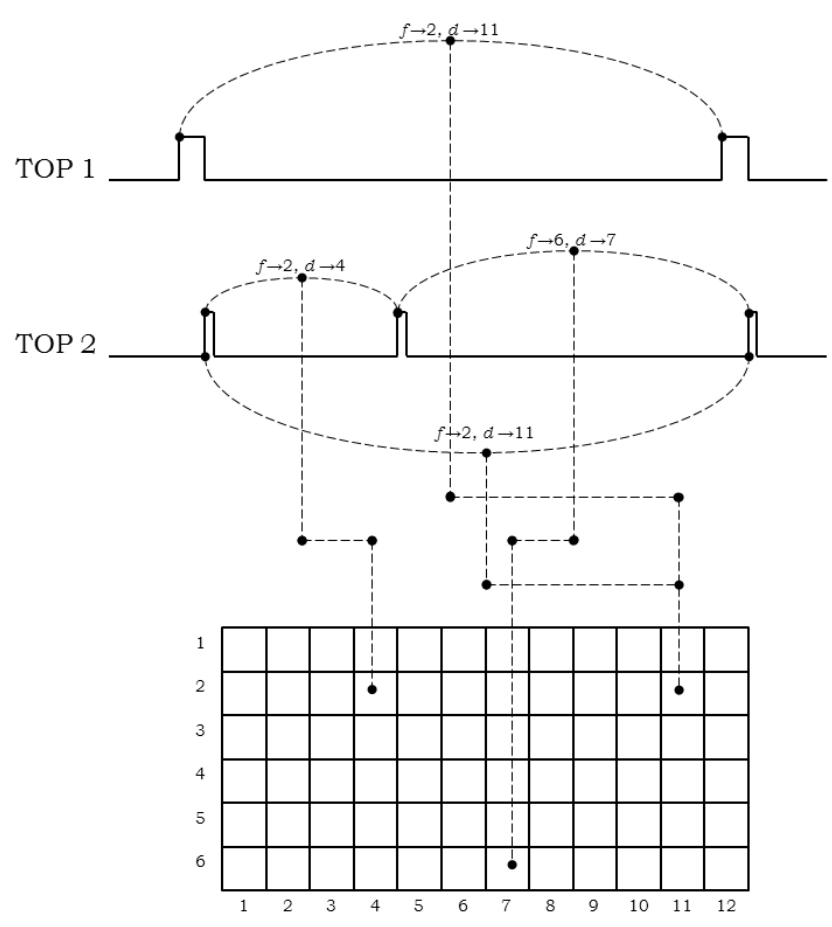

Figure 5: Temporal recurrence hashing.

for a pair of two identical CF segments with a length of 15 seconds, the corresponding $h\left(f_{i, j} ; d_{i, j}\right)$ should be equal to $15 \times 29.97 \approx 450$, where 29.97 is the frame rate of the video. The hashing histogram is thus built by scanning all frame pairs in each B-TOP.

$$
h\left(f_{i, j} ; d_{i, j}\right)=h\left(f_{i, j} ; d_{i, j}\right)+\min \left(l_{i}, l_{j}\right)
$$

Note that we do not really insert the peak pairs into the hash table because this processing requires a huge amount of memory usage. Instead, we scan all B-TOPs, and for all peak pairs in each B-TOP, the algorithm performs histogram voting based on $f$ and $d$ of each pair to build the recurrence hashing histogram. We discard B-TOPs that contain an excessively large number of peaks. Such B-TOPs usually result from segments of self-similar sequences, e.g. a baseball game, and cause a large computational burden and memory usage. According to our experiments, a threshold $\delta_{1}$ of 45 per 10-hour period is a good choice.

\subsection{Local Maximum Detection}

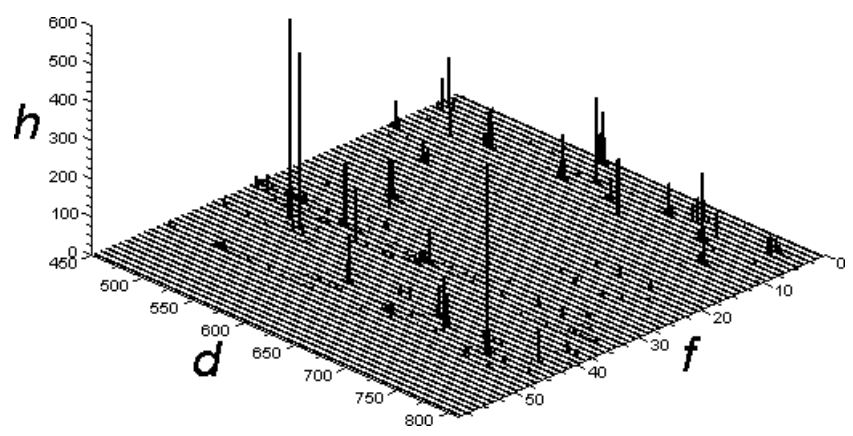

Figure 6: 2D view of a recurrence hashing histogram. $f \rightarrow$ min.; $d \rightarrow$ sec.; $h \rightarrow h(f, d)$ 


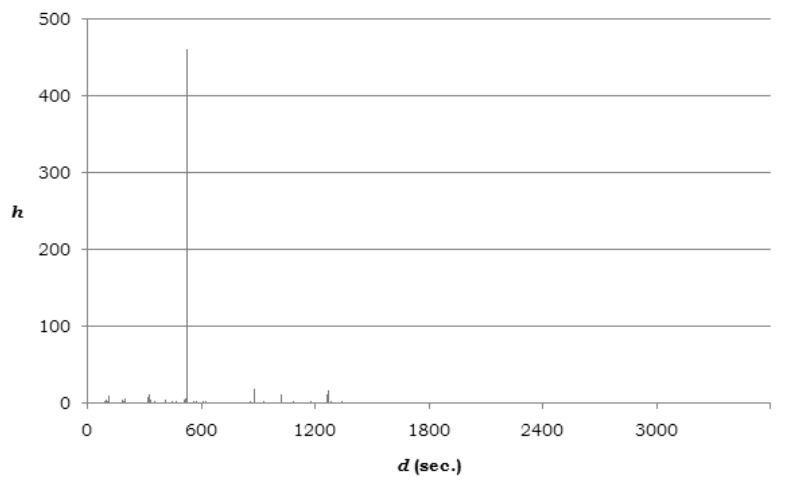

(a) $f=37$ (min.)

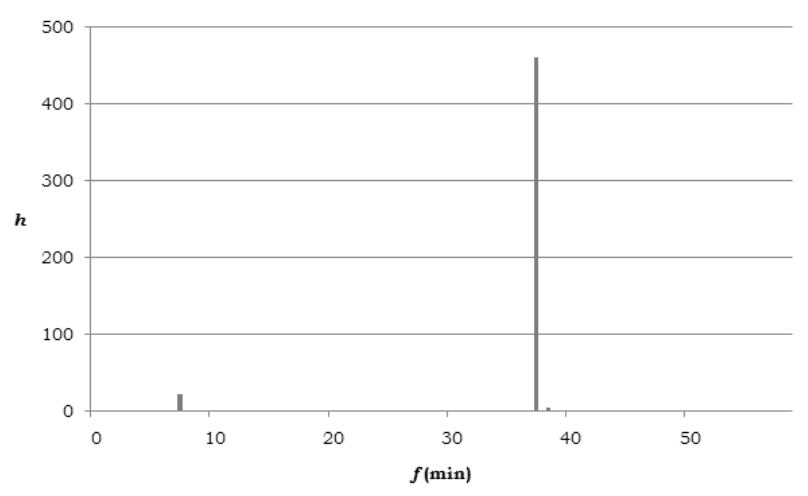

(b) $d=522$ (sec.)

Figure 7: 1D view of the histogram shown in Figure 6.

Figure 6 and 7 show an example of the recurrence hashing histogram built from a 1-hour sequence. For convenience, we only show a part of the whole 2-dimensional histogram in Figure 6. On the other hand, Figure 7 show two planes (Figure 7a and 7b) extracted from the whole histogram. By observing the hashing histogram, we found that the peak pairs derived from a CF segment pair usually form a local maximum. This is because most noise peak pairs, in which the two peaks are non-identical but mapped to the same $f$ and $d$, are near-uniformly distributed in the bins of the recurrence hash table. As a result, the values of these bins are usually far lower than those derived from the CF segments. Based on this observation, we detect the local maximums from the hashing histogram. For each local maximum, the peak pairs, which are inserted into the bin corresponding to this local maximum, are assembled. By doing so, we can detect the existence of CFs, assemble the discrete peaks into a whole $\mathrm{CF}$ segment, and further detect the pairwise relationship between identical CF segments.

To detect local maximums, a $3 \times 3$ window is cast on each bin, and the value of the central bin $\left(f_{c}, d_{c}\right)$ is compared with those of the eight neighboring bins. The detected candidate local maximums are put through a noise filter. We calculate the sum $s_{c}$ of the number of overlapping frames in all nine bins (Eq. 7). Theoretically, for a pair of identical CF segments with a length of 15 seconds, the corresponding $s_{c}$ should be equal to or more than $15 \times 29.97 \approx 450$. The reason of using the sum $s_{c}$ instead of the single value of the central bin is because the resolution of $f$ is minute so that some CF segments may reside in the position of minute boundaries. In this case, the peak pairs will be inserted into multiple neighboring bins instead of a single bin. We set a threshold $\delta_{\min }=10 \times 29.97 \approx 300$ to filter out noise because Japanese CFs are 15 seconds or longer.

$$
s_{c}=\sum_{f=f_{c}-1}^{f_{c}+1} \sum_{d=d_{c}-1}^{d_{c}+1} h(f ; d)
$$

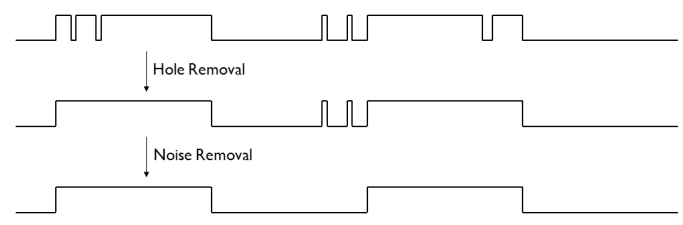

Figure 8: Morphological filtering.

Given a detected local maximum, all peak pairs in the corresponding bin are assembled and concatenated to form a Temporal Occurrence Pattern, or M-TOP (Maximum-TOP, Figure 8). Each M-TOP contains multiple candidate CF segments and corresponds to one local maximum. Note that we did not really build the recurrence hash table because of the huge amount of memory it would have taken. Instead, we scan all B-TOPs, and for all peak pairs in each B-TOP, the algorithm calculates the corresponding $f$ and $d$, and then assembles the peak pairs if $(f, d)$ is in the set of detected local maximums. Morphological filtering is further performed to remove noises from each M-TOP (Figure 8). If the interval between two consecutive peaks is shorter than a threshold $\delta_{2}$, these peaks are merged into one peak. If the length of a peak is shorter than $\delta_{\min }=300$, it is discarded as a noise.

\subsection{Post-Processing}

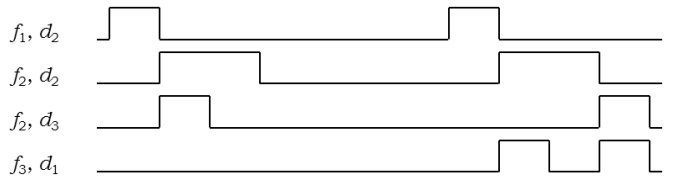

(a) M-TOPs.

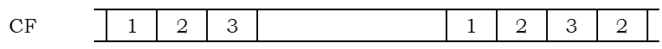

(b) Candidate CF segments.

Figure 9: CF detection.

The algorithm projects all boundaries derived from the M-TOPs (Figure 9a) to the video sequence. The sequence is thus partitioned into multiple candidate CF segments (Figure $9 \mathrm{~b})$. We use two thresholds, $\delta_{\min }$ and $\delta_{\max }$, to represent the restricted duration of Japanese CFs and filter out nonCF segments. $\delta_{\min }=300$ was also used for local maximum detection and noise removal (Section 4.3). Because the maximum length of Japanese CFs is 30 seconds, $\delta_{\max }$ is set to $35 \times 29.97 \approx 1,049$ with 29.97 being the frame rate. The pairwise relationship between $\mathrm{CF}$ segments can be derived on the basis of the parameter $d$ of the M-TOPs. Transitive closure is then performed to the set of $\mathrm{CF}$ segment pairs to group them into CF clusters. In broadcast video, two different CFs may be continuously broadcasted several times. In this case, the peak pairs derived from them will have the same temporal distribution ( $f$ and $d$ ), and be merged to form a long candidate CF segment (see the M-TOP $\left(f_{2}, d_{2}\right)$ in Figure 9a which corresponds to consecutive occurrence of $\mathrm{CF} 2$ and $\mathrm{CF} 3$ ). This problem however can be easily avoided if either of these two CFs has been individually broadcasted in other positions of the same sequence (see the third segment of CF 2 in Figure 9). 


\section{EXPERIMENTATION AND EVALUATION}

\subsection{Experimental Setting}

We tested our algorithm using a large-scale broadcast video archive comprised of two sequences, A10H and A1M, broadcasted from a Japanese TV station. $\mathrm{A} 10 \mathrm{H}$ is a 10-hour sequence and $\mathrm{A} 1 \mathrm{M}$ is a $\mathbf{1 - m o n t h}$ sequence. A10H contains $66 \mathrm{CF}$ clusters and $202 \mathrm{CF}$ segments, which were manually annotated to generate the ground truth. Because it is almost impossible to manually annotate a 1-month sequence because of the huge amount of data it contains, we used A1M only for the evaluation of efficiency. We used a Linux server with an Intel Xeon $2.66 \mathrm{GHz}$ CPU and $128 \mathrm{~GB}$ of main memory. We evaluated our algorithm in terms of two criteria: detection and localization.

We use $\mathbf{R}=\left\{\mathbf{r}_{1}, \mathbf{r}_{2}, \cdots, \mathbf{r}_{m}\right\}$ to denote our detection results, where $\mathbf{r}$ indicates the detected CF cluster and $m$ is the number of clusters. We also use $\mathbf{G}=\left\{\mathbf{g}_{1}, \mathbf{g}_{2}, \cdots, \mathbf{g}_{n}\right\}$ to denote the ground truth, where $\mathbf{g}$ indicates the CF cluster and $n$ is the number of clusters in ground truth set. Thus, the detection accuracy can be evaluated by using precision $\mathcal{P}_{d}$ (Eq. 8) and recall $\mathcal{R}_{d}$ (Eq. 9), where $|\cdot|$ indicates the number of segments.

$$
\begin{aligned}
\mathcal{P}_{d} & =\frac{\sum_{i=1}^{m} \max _{j=1}^{n}\left|\mathbf{r}_{i} \cap \mathbf{g}_{j}\right|}{\sum_{i=1}^{m}\left|\mathbf{r}_{i}\right|} \\
\mathcal{R}_{d} & =\frac{\sum_{i=1}^{m} \max _{j=1}^{n}\left|\mathbf{r}_{i} \cap \mathbf{g}_{j}\right|}{\sum_{j=1}^{n}\left|\mathbf{g}_{j}\right|}
\end{aligned}
$$

The localization accuracy is used to evaluate how exactly the algorithm can identify the beginning and end frames of a CF segment. To do this, we only focus on true positive (TP) segments, i.e. false alarms and misses are discarded. We respectively use $\mathbf{R}^{*}=\left\{r_{1}, r_{2}, \cdots, r_{o}\right\}$ and $\mathbf{G}^{*}=$ $\left\{g_{1}, g_{2}, \cdots, g_{o}\right\}$ to denote our results and the ground truth. $r$ indicates the TP segments in the result sets and $g$ indicates those in the ground truth set. Precision and recall are defined as follows, where $|\cdot|$ indicates the number of frames.

$$
\begin{gathered}
\mathcal{P}_{l}=\frac{\sum_{i=1}^{o}\left|r_{i} \cap g_{i}\right|}{\sum_{i=1}^{o}\left|r_{i}\right|} \\
\mathcal{R}_{l}=\frac{\sum_{i=1}^{o}\left|r_{i} \cap g_{j}\right|}{\sum_{i=1}^{o}\left|g_{i}\right|}
\end{gathered}
$$

We used four parameters including the maximum number of peaks in each B-TOP, $\delta_{1}=45$ (Section 4.2 ), the minimum interval used for merging peaks, $\delta_{2}=6$ (Section 4.3), and the restricted duration of CF segments, $\delta_{\min }=300$ (Section 4.3 and 4.4) and $\delta_{\max }=1,049$ (Section 4.4). $\delta_{1}$ and $\delta_{2}$ were set based on experimentation.

\subsection{Experimental Results}

We used two state-of-the-art techniques as baselines, a hashing-based algorithm [4] and a clustering-based algorithm [2]. More details on the two reference techniques can be found in Section 2. Table 1 compares the performance of our algorithm (DSTRH) with those of the two baselines. A10H was used. $\mathcal{T}$ indicates the processing time of $\mathrm{CF}$ detection and localization. We can see that DSTRH outperformed the baselines for all criteria, i.e. detection $\left(\mathcal{F}_{d}\right)$, localization $\left(\mathcal{F}_{l}\right)$, and efficiency $(\mathcal{T})$. All false alarms detected by the three algorithms are either duplicates or near-duplicates. Duplicates result from rebroadcast non-CF segments, while near-duplicates result from self-similar programs, e.g. announcer scenes, scenes of the question period in the Japanese parliament, or sports games. All misses of DSTRH are due to variations in the time display between CF segments. The luminance-based fingerprinting strategy (Section 4.1) of DSTRH is sensitive to this kind of slight variation. A more robust fingerprinting strategy can be used to solve this problem, and this will be a future work. On the other hand, our algorithm performed CF detection and identification on a 10-hour sequence within four seconds, which is far faster than the two baselines.

\subsection{Experiment on 1-Month Archive}

We applied DSTRH to the 1-month archive A1M. The corresponding processing time is 87 minutes. The most timeconsuming processes include temporal recurrence hashing (18 minutes), local maximum detection (27 minutes), and segment concatenation (37 minutes). One obvious reason for the large increase in processing time relative to $\mathrm{A} 10 \mathrm{H}$ is the large increase in archive scale. Another important reason concerns the usage of cache memory. In our algorithm, a large fingerprint-based hash table is built during the temporal occurrence hashing stage (Section 4.1). The cache of the Linux server used for experimentation is $16 \mathrm{MB}$. In the case of $\mathrm{A} 10 \mathrm{H}$, the size of the hash table is still small enough so that it can be stored in the cache. However, the memory required for storing the hash table of $\mathrm{A} 1 \mathrm{M}$ is more than 100 $\mathrm{MB}$ so that further scanning processes require frequent data accesses, data transfers, and cache replacements between the cache and physical memory. Even so, CF detection and identification on the 1-month sequence was completed within 87 minutes, which is extremely fast. Thus, CF mining on a 1-year archive can be achieved within $(87 \times 12)=17 \mathrm{hr}$. \& 24 min. $<18$ hr.

From A1M, our algorithm detected 3,875 CF clusters containing a total of $28,812 \mathrm{CF}$ segments. By observing the detection results, we found that CFs of different categories (cosmetic, food, beverage, DVD, etc.) are usually broadcasted in different time slots. Figure 10 show an example. The two dimensions shown in Figure 10b and 10c correspond to the airdate and the airtime. From the temporal distribution of these two CFs, we can see that in most cases the food $\mathrm{CF}$ is broadcasted in the daytime $(10: 00 \sim 20: 00)$, while the film $\mathrm{CF}$ is more frequently broadcasted in the nighttime (00:00 06:00). This is because in Japan, the daytime is the most popular time slot for housewives, while the nighttime is more popular for audiences from teens to thirties. If we extend these graphs to a 1-year archive and multiple channels, we can generate a more informative 3-dimensional point diagram. One of our future tasks will be to apply data mining techniques to the CF clusters based on this 3-dimensional point diagram to investigate the relation between the temporal distribution and the semantic characteristics of CFs.

\section{CONCLUSION}

This paper described a novel dual-stage temporal recurrence hashing algorithm for detection and identification of commercial films (CFs) in a large-scale broadcast video archive. This algorithm is fully unsupervised, generic, and super-fast. In experiments the algorithm's accuracies of detection $\left(\mathcal{F}_{d}\right)$ and localization $\left(\mathcal{F}_{l}\right)$ were $90.59 \%$ and $98.06 \%$. The algorithm is also very efficient. CF detection and identification on a 10-hour and a 1-month sequence respectively took only four seconds and $\mathbf{8 7}$ minutes. Even without parallel computation, our algorithm would take less than 18 hours to perform $\mathrm{CF}$ detection, localization, and identification on an archive containing a whole year's worth of video. We used a luminance-based fingerprinting strategy for image hashing. In the future, we will apply our algorithm to other fingerprinting strategies to investigate whether it is invariant to different image hashing techniques. We are also planning to extend our algorithm to the audio domain through the use of acoustic fingerprinting strategies. 
Table 1: Performance comparison.

\begin{tabular}{|l|c|c|c|c|c|c|c|}
\hline Algorithm & $\mathcal{P}_{d}(\%)$ & $\mathcal{R}_{d}(\%)$ & $\mathcal{F}_{d}(\%)$ & $\mathcal{P}_{l}(\%)$ & $\mathcal{R}_{l}(\%)$ & $\mathcal{F}_{l}(\%)$ & $\mathcal{T}$ (sec.) \\
\hline DSTRH & 93.19 & 88.12 & 90.59 & 99.13 & 97.01 & 98.06 & 4 \\
\hline Dohring [4] & 71.26 & 89.60 & 79.38 & 98.70 & 96.78 & 97.73 & 128 \\
\hline Berrani [2] & 72.22 & 83.66 & 77.52 & 99.18 & 83.47 & 90.65 & 37 \\
\hline
\end{tabular}

\section{REFERENCES}

[1] A. Albiol, M. J. Fulla, A. Albiol, and L. Torres. Detection of tv commercials. In Proceedings of the IEEE International Conference on Acoustics, Speech, and Signal Processing, pages 541-544, 2004.

[2] S.-A. Berrani, G. Manson, and P. Lechat. A non-supervised approach for repeated sequence detection in tv broadcast streams. Image Commun., 23(7):525-537, 2008.

[3] M. Covell, S. Baluja, and M. Fink. Advertisement detection and replacement using acoustic and visual repetition. In Proceedings of the 8th IEEE International Workshop on Multimedia Signal Processing, pages 461-466, 2006.

[4] I. Döhring and R. Lienhart. Mining tv broadcasts for recurring video sequences. In CIVR '09: Proceeding of the ACM International Conference on Image and Video Retrieval, pages 1-8, 2009.

[5] L.-Y. Duan, J. Wang, Y. Zheng, J. S. Jin, H. Lu, and C. $\mathrm{Xu}$. Segmentation, categorization, and identification of commercial clips from tv streams using multimodal analysis. In MULTIMEDIA '06: Proceedings of the 14th annual ACM international conference on Multimedia, pages 201-210, 2006.

[6] J. M. Gauch and A. Shivadas. Identification of new commercials using repeated video sequence detection. In ICIP, pages 1252-1255, 2005

[7] J. M. Gauch and A. Shivadas. Finding and identifying unknown commercials using repeated video sequence detection. Comput. Vis. Image Underst., 103(1):80-88, 2006.

[8] A. G. Hauptmann and M. J. Witbrock. Story segmentation and detection of commercials in broadcast news video. In $A D L$ '98: Proceedings of the Advances in Digital Libraries Conference, pages 168-179, 1998.

[9] C. Herley. Argos: automatically extracting repeating objects from multimedia streams. IEEE Transactions on Multimedia, 8(1):115-129, 2006.

[10] X.-S. Hua, L. Lu, and H.-J. Zhang. Robust learning-based tv commercial detection. In ICME, pages 149-152, 2005.

[11] Y. Li, J. S. Jin, and X. Zhou. Matching commercial clips from tv streams using a unique, robust and compact signature. In DICTA '05: Proceedings of the Digital Image Computing on Techniques and Applications, pages 355-362, 2005.

[12] Y. Li, D. Zhang, X. Zhou, and J. S. Jin. A confidence based recognition system for tv commercial extraction. In $A D C$ '08: Proceedings of the nineteenth conference on Australasian database, pages 57-64, 2008.

[13] R. Lienhart, C. Kuhmunch, and W. Effelsberg. On the detection and recognition of television commercials. In ICMCS '97: Proceedings of the 1997 International Conference on Multimedia Computing and Systems, pages 435-445, 1997.

[14] A. Shivadas and J. M. Gauch. Real-time commercial recognition using color moments and hashing. In $C R V$ '07: Proceedings of the Fourth Canadian Conference on Computer and Robot Vision, pages 465-472, 2007.
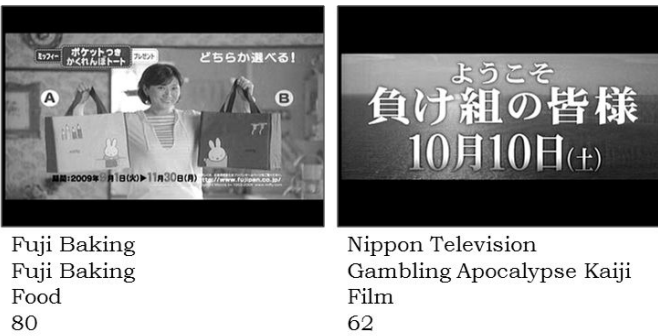

(a) Example of a food $\mathrm{CF}$ and a film $\mathrm{CF}$.

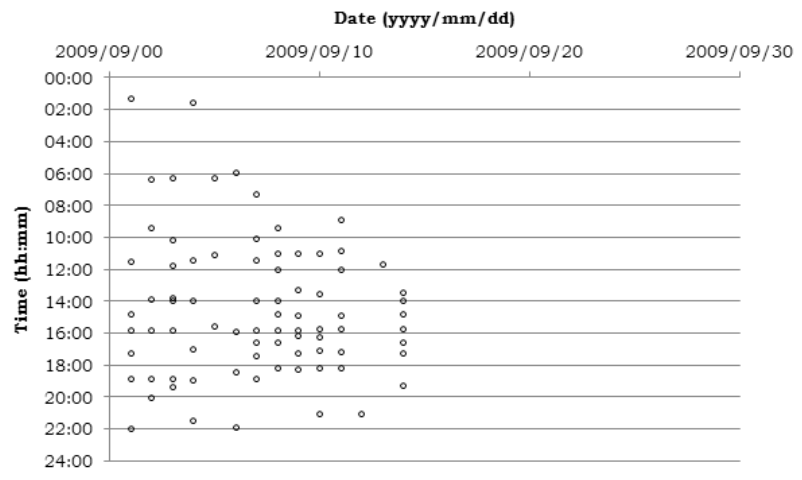

(b) Temporal distribution of the food CF.

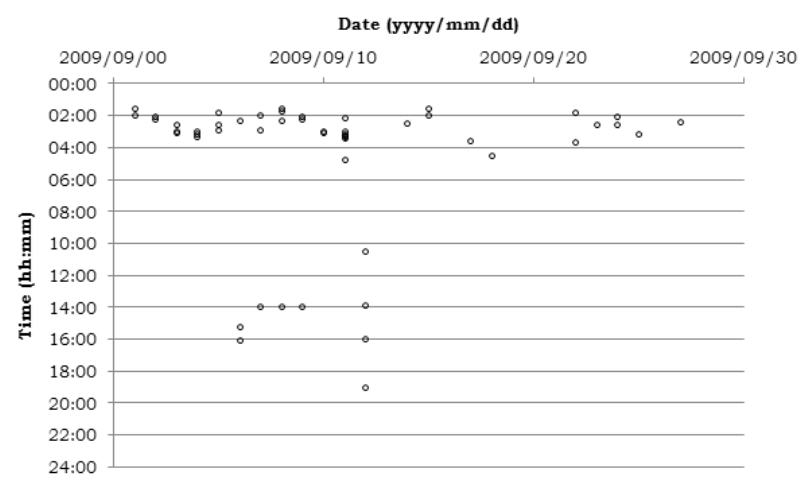

(c) Temporal distribution of the film CF.

Figure 10: Temporal distribution of two CFs. 